\title{
Experimental asymmetric phase-covariant quantum cloning of polarization qubits
}

\author{
Jan Soubusta, ${ }^{1}$ Lucie Bartůšková, ${ }^{2}$ Antonín Černoch, ${ }^{1}$ Miloslav Dušek, ${ }^{2}$ and Jaromír Fiurášek ${ }^{2}$ \\ ${ }^{1}$ Joint Laboratory of Optics of Palacky University and Institute of Physics of Academy of Sciences of the Czech Republic, \\ 17. listopadu 50A, 77907 Olomouc, Czech Republic \\ ${ }^{2}$ Department of Optics, Faculty of Science, Palacký University, \\ 17. listopadu 50, 77200 Olomouc, Czech Republic
}

(Dated: October 29, 2018)

\begin{abstract}
We report on two optical realizations of the $1 \rightarrow 2$ asymmetric phase-covariant cloning machines for polarization states of single photons. The experimental setups combine two-photon interference and tunable polarization filtering that enables us to control the asymmetry of the cloners. The first scheme involves a special unbalanced bulk beam splitter exhibiting different splitting ratios for vertical and horizontal polarizations, respectively. The second implemented scheme consists of a balanced fiber coupler where photon bunching occurs, followed by a free-space part with polarization filters. With this later approach we were able to demonstrate very high cloning fidelities which are above the universal cloning limit.
\end{abstract}

PACS numbers: 03.67.-a, 03.67.Hk, 42.50.-p

\section{INTRODUCTION}

Optimal copying of quantum states is an important primitive in quantum information processing [1, 2]. Since exact copying of unknown quantum states is forbidden due to the linearity of quantum mechanics [3] this task can be accomplished only approximately. A figure of merit commonly employed to quantify the performance of quantum cloners is the fidelity of the clones [1, 2]. Optimal cloning machines that maximize the cloning fidelity have been identified theoretically for a wide range of classes of input states and numbers of copies [1, 2]. The universal quantum cloners copy all states from the underlying Hilbert space with the same fidelity [4, 5, 6, 7]. Sometimes, however it is more beneficial to clone optimally only a certain subset of states. A particularly important example is the phase-covariant quantum cloner [8, 9, 10, 11] that optimally copies all qubits from the equator of the Bloch sphere, i.e. all balanced superpositions of the computational basis states. The advantage of such dedicated cloning machine is that it reaches higher cloning fidelities than the universal machine.

Phase-covariant cloning represents an optimal individual eavesdropping attack on BB84 quantum key distribution protocol [12, 13]. In this context, the asymmetric cloning machines that produce two copies with different fidelities [13, 14, 15] are particularly important. Tuning the asymmetry of the cloning operation enables to control the trade-off between information on a secret cryptographic key gained by the eavesdropper and the amount of noise added to the copy which is sent down the channel to the authorized receiver.

For potential applications in quantum communication, such as eavesdropping on quantum key distribution, cloning of the quantum states of single photons is of great interest [1, 2]. Universal cloning of polarization states of single photons has been implemented experimentally using either stimulated parametric downconversion [16, 17] or bunching of photons on a balanced beam splitter [18, 19, 20]. Asymmetric universal cloning 21] and symmetric $1 \rightarrow 3$ phase-covariant cloning [22] of photonic qubits have also been realized. Recently, we have experimentally demonstrated the optimal symmetric $1 \rightarrow 2$ phase-covariant cloning of the polarization states of single photons [23, 24]. We have also implemented an all-fiber setup for optimal phase-covariant asymmetric cloning of qubits represented by single photons that can simultaneously propagate in two distinct single-mode optical fibers [25].

In the present paper we report on the experimental demonstration of the optimal asymmetric phasecovariant cloning of the polarization states of single photons. In contrast to our previous fiber-optics experiment our present approach does not rely on single-photon interference and we therefore do not have to stabilize any first-order interferometer. We have implemented two schemes both of which are extensions of setups utilized previously for symmetric phase-covariant cloning as described in Refs. 23, 24].

The first setup involves an interference of two photons (signal, and an ancilla in a vertical polarization state) on a specially tailored unbalanced beam splitter, which ideally affects the optimal symmetric phasecovariant cloning operation [11, 23]. The cloner is then made asymmetric by applying partial polarization filters to both clones. This filtering is realized by means of tilted glass plates, introducing different transmittances of the TE and TM polarization modes according to Fresnel equations. A second approach relies on the combination of optimal universal cloning and polarization filtration. The former is achieved by an interference of the two photons on a balanced beam splitter [18, 19, 20]. We utilize advantageously a fiber coupler which allows us to reach very high visibility of the Hong-Ou-Mandel interference [26]. The two clones are then subjected to partial polarization filtration such that the output of the machine corresponds to that of the optimal phase-covariant cloner. With this latter scheme we observe cloning fideli- 
ties exceeding those achievable with any optimal universal cloning machine. We thus clearly demonstrate the advantage of phase-covariant asymmetric quantum cloners over the universal asymmetric cloner in cases of restricted sets of input states.

The paper is structured as follows. In Section II we briefly review the theory of the optimal asymmetric phase-covariant cloning operations. In Section [II] we present the experimental implementation of the asymmetric cloner that relies on the two-photon interference on an unbalanced beam splitter followed by polarization filtration which tunes the asymmetry of the cloner. In Section [V] we discuss an alternative scheme consisting of a sequence of the optimal universal cloner based on photon bunching in a balanced fiber coupler followed again by appropriate polarization filtration. Finally, section $\mathrm{V}$ contains a brief summary of the main results and a comparison of the two schemes.

\section{OPTIMAL PHASE-COVARIANT CLONING}

We are interested in copying of a polarization state of a single photon $|\psi\rangle$. This single-qubit state can be conveniently parametrized by two Euler angles $\theta$ and $\phi$,

$$
|\psi\rangle=\cos \frac{\theta}{2}|V\rangle+e^{i \phi} \sin \frac{\theta}{2}|H\rangle .
$$

Here the two orthogonal computational basis states $|V\rangle$ and $|H\rangle$ represent the vertical and horizontal linear polarization states, respectively. In this paper we focus on the cloning of the polarization states situated on the equator of the Bloch sphere $\left(\theta=\frac{\pi}{2}\right)$,

$$
|\psi\rangle=\frac{1}{\sqrt{2}}\left(|V\rangle+e^{i \phi}|H\rangle\right) .
$$

The optimal asymmetric phase-covariant cloning transformation reads [25],

$$
\begin{aligned}
& |V\rangle|V\rangle \rightarrow|V\rangle|V\rangle, \\
& |H\rangle|V\rangle \rightarrow \sqrt{q}|V\rangle|H\rangle+\sqrt{1-q}|H\rangle|V\rangle,
\end{aligned}
$$

where $q \in[0,1]$ is the asymmetry parameter. Note that this unitary transformation requires only two qubits, the signal whose state we want to clone and an ancilla qubit (a blank copy) prepared in a fixed state $|V\rangle$. The second line of Eq. (3) means creation of a superposition of the input state with a state where the two photons have been exchanged. Such states are naturally produced by a beam splitter with splitting ratio depending on the asymmetry parameter $q$.

The quality of the clones is quantified by their fidelity, which is defined as the overlap of each clone state with the original state (2). The fidelities of the two clones produced by the optimal asymmetric phase-covariant cloning transformation (3) read,

$$
F_{1}=\frac{1}{2}(1+\sqrt{1-q}), \quad F_{2}=\frac{1}{2}(1+\sqrt{q}) .
$$

In the case of symmetric cloning $(q=1 / 2)$ both fidelities have the same value $F_{\mathrm{sym}, \mathrm{pc}} \approx 85.4 \%$. For comparison we give also the clone fidelities achievable by the optimal universal asymmetric cloning [2],

$$
F_{\mathrm{u} 1}=1-\frac{(1-p)^{2}}{2\left(1-p+p^{2}\right)}, \quad F_{\mathrm{u} 2}=1-\frac{p^{2}}{2\left(1-p+p^{2}\right)},
$$

where parameter $p \in[0,1]$ controls the asymmetry of the two clones. An universal cloner copies all states (not only the equatorial ones) with the same fidelities $F_{\mathrm{u} 1}$ and $F_{\mathrm{u} 2}$. The fidelity of the symmetric universal cloner $(p=1 / 2)$ reads $F_{\text {sym,univ }} \approx 83.3 \%[4,[5]$.

\section{FREE SPACE REALIZATION WITH A SPECIAL BEAM SPLITTER}

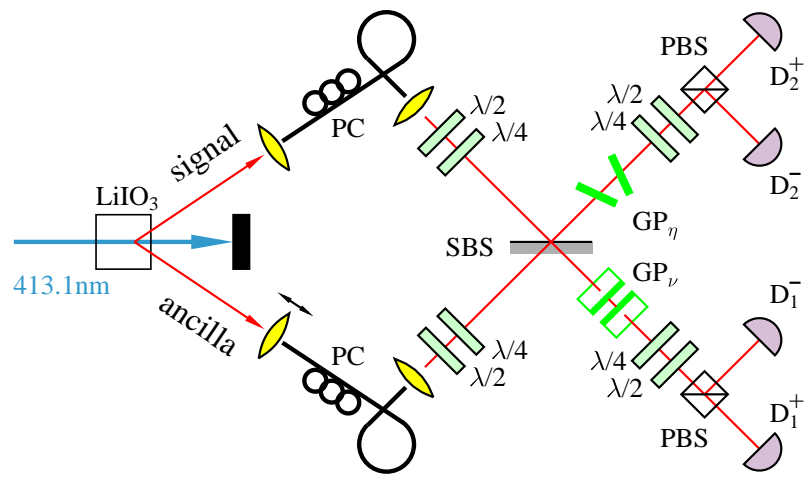

FIG. 1: (Color online) Scheme of the cloning setup based on the special beam splitter and polarization dependent losses. $\mathrm{PC}$ - polarization controller, SBS - special beam splitter, $\mathrm{GP}_{\eta}$, $\mathrm{GP}_{\nu}$ - polarization dependent losses, PBS - polarizing cube beam splitter, $\lambda / 2, \lambda / 4$ - wave plates, $\mathrm{D}$ - detector.

The first setup for the optimal asymmetric phasecovariant cloning of polarization states of single photons is shown in Fig. 1. This setup is based on an interference of two photons on a special unbalanced beam splitter (SBS) with splitting ratios different for vertical and horizontal polarizations. The interference on SBS is followed by polarization filtration performed on each output port of the beam splitter. The polarization filters are realized by tilted glass plates (GP), where the tilt angle determines the ratio of transmittances for the horizontal and vertical polarizations. As we shall show below, by tilting the plates we are able to control the asymmetry of the cloner. The device operates in the coincidence basis and successful cloning is heralded by the presence of a single photon in each output port of the cloning machine. In practice, we postselect only the cases when we observe coincidence between photon detections in the upper and lower output arms. All other events are discarded.

Let us describe the experimental setup in more details. A non-linear crystal of $\mathrm{LiIO}_{3}$ is pumped by cw $\mathrm{Kr}^{+}$laser 
at $413 \mathrm{~nm}$ to produce pairs of photons in the type I process of spontaneous parametric down conversion. Photons comprising each pair exhibit tight time correlations and are horizontally polarized. The photons are coupled into single mode fibers that serve as spatial filters. The polarization controllers (PC) are adjusted such as to ensure horizontal linear polarization of the two photons at the outputs of the fibers. The polarization state of each photon is set by means of half- and quarter-wave plates $(\lambda / 2, \lambda / 4)$. The photon in the upper arm represents the signal qubit whose state should be cloned. The other photon serves as the ancilla and its polarization state is fixed to $|V\rangle$, c.f. Eq. (3). Both photons enter the special beam splitter which forms the Hong-Ou-Mandel interferometer [26]. The pairs of tilted glass plates introduce different amplitude transmittances for horizontal and vertical polarizations, $\left(\eta_{V}, \eta_{H}\right.$ for $\mathrm{GP}_{\eta}$; and $\nu_{V}, \nu_{H}$ for $\left.\mathrm{GP}_{\nu}\right)$. It is convenient to define the intensity transmittance ratios,

$$
\Sigma_{\eta}=\left(\frac{\eta_{V}}{\eta_{H}}\right)^{2}, \quad \Sigma_{\nu}=\left(\frac{\nu_{V}}{\nu_{H}}\right)^{2}
$$

$\mathrm{GP}_{\eta}$ dominantly attenuates vertical polarization, hence $\Sigma_{\eta} \leq 1$, while $\mathrm{GP}_{\nu}$ imposes higher losses for horizontal polarization, and $\Sigma_{\nu} \geq 1$. We use two glass plates in each arm to reach higher transmittance ratios for the two polarizations. Moreover, since the two plates are tilted in opposite directions, the beams are not transversally displaced by the filtration.

The transformation introduced by the setup shown in Fig. 1 can be written in the form

$$
\begin{aligned}
|V\rangle_{\text {sig }}|V\rangle_{\text {anc }} \rightarrow & \eta_{V} \nu_{V}\left(r_{V}^{2}-t_{V}^{2}\right)|V\rangle_{1}|V\rangle_{2}, \\
|H\rangle_{\text {sig }}|V\rangle_{\text {anc }} \rightarrow & \eta_{H} \nu_{V} r_{V} r_{H}|V\rangle_{1}|H\rangle_{2} \\
& -\eta_{V} \nu_{H} t_{V} t_{H}|H\rangle_{1}|V\rangle_{2},
\end{aligned}
$$

where $r_{V}, r_{H} ; t_{V}, t_{H}$ are the (fixed) real amplitude reflectances and transmittances of the SBS. We use notation $R_{j}=r_{j}^{2}$ and $T_{j}=t_{j}^{2}, j=H, V$, for intensity reflectances and transmittances and we have $R_{j}+T_{j}=1$ for a lossless beam splitter.

Mapping (7) becomes equivalent to the unitary cloning transformation (3) up to an overall prefactor representing the amplitude of the probability of success of the cloning if the following two conditions are satisfied simultaneously,

$$
\begin{aligned}
\eta_{H} \nu_{V} r_{V} r_{H} & =\sqrt{q} \eta_{V} \nu_{V}\left(r_{V}^{2}-t_{V}^{2}\right) \\
-\eta_{V} \nu_{H} t_{V} t_{H} & =\sqrt{1-q} \eta_{V} \nu_{V}\left(r_{V}^{2}-t_{V}^{2}\right)
\end{aligned}
$$

After some algebra we obtain the transmittance ratios of the polarization filters expressed as functions of the asymmetry parameter $q$,

$$
\begin{aligned}
\Sigma_{\eta} & =\frac{R_{V} R_{H}}{\left(2 R_{V}-1\right)^{2}} \frac{1}{q}, \\
\Sigma_{\nu} & =\frac{\left(1-R_{V}\right)\left(1-R_{H}\right)}{\left(2 R_{V}-1\right)^{2}} \frac{1}{1-q} .
\end{aligned}
$$

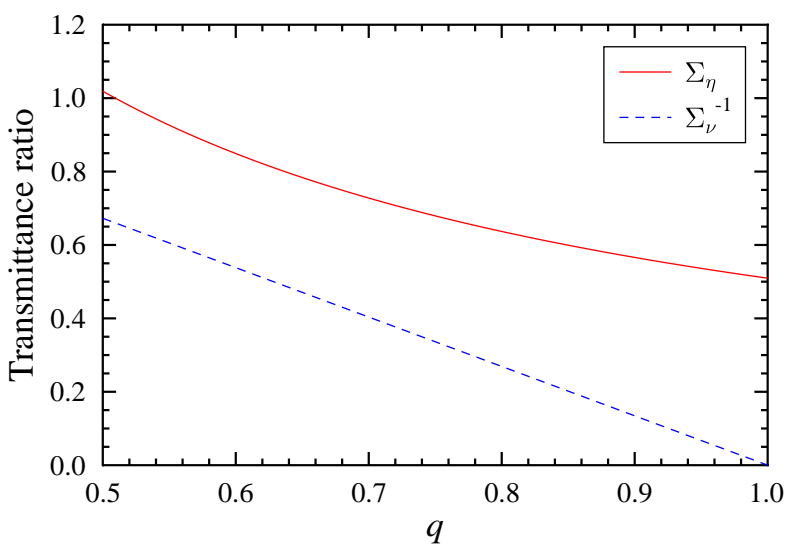

FIG. 2: (Color online) Transmittance ratios $\Sigma_{\eta}$ and $\Sigma_{\nu}^{-1}$ for the asymmetric cloner with the special unbalanced beam splitter. Plotted dependences were calculated according to Eq. (9) using experimentally determined parameters of SBS: $R_{V}=75.8 \%$ and $R_{H}=17.9 \%$.

We have chosen the splitting ratios of the SBS such that symmetric cloning could be realized without any further polarization filtration. If we set $\Sigma_{\eta}=\Sigma_{\nu}=1$ and $q=1 / 2$ in Eq. (9) we obtain $R_{V}=\frac{1}{2}\left(1+\frac{1}{\sqrt{3}}\right) \approx 78.9 \%$ and $R_{H}=1-R_{V}$ [23]. The experimentally determined parameters of the custom-made SBS manufactured by Ekspla read $R_{V}=75.8 \%$ and $R_{H}=17.9 \%$ which is close to the desired values. Figure 2 shows the theoretical dependence of the transmittance ratios on $q$ calculated according to Eq. (9) using the experimentally determined values of $R_{H}$ and $R_{V}$. In particular, note that the symmetric operation would be achieved for $\Sigma_{\nu}^{-1}=0.67$ and $\Sigma_{\eta}=1.02$. The probability of success of the cloning is given by $P_{\text {succ }}^{\mathrm{SBS}}=\eta_{V}^{2} \nu_{V}^{2}\left(r_{V}^{2}-t_{V}^{2}\right)^{2}$. It can be shown that $P_{\text {succ }}$ is highest for the symmetric cloner and decreases with increasing asymmetry, because the losses introduced by polarization filters are increasing. Comparison of the measured and theoretically attainable $P_{\text {succ }}$ is given at the end of Sec. IV for both setups.

As we already mentioned, the cloning procedure is successful only if there is one photon in each output arm of the device. The performance of the cloning machine is probed by polarization analysis of the two clones. The setting of wave plates at the output is inverse with respect to the signal photon preparation. This means that the photons with the same polarization as the signal photon are transmitted through the PBS to the detector $\mathrm{D}^{+}$whereas the photons with orthogonal polarization are reflected to the detector $\mathrm{D}^{-}$. This allows us to infer the cloning fidelities from the four measured coincidence rates $C^{ \pm \pm}$between detectors at the two output arms. For instance, coincidence rate $C^{++}$represents the number of simultaneous clicks of detectors $\mathrm{D}_{1}^{+}$and $\mathrm{D}_{2}^{+}$per second and the other coincidence rates are determined similarly. The fidelities of the clones are calculated as the ratio of coincidences corresponding to the projection of the first 


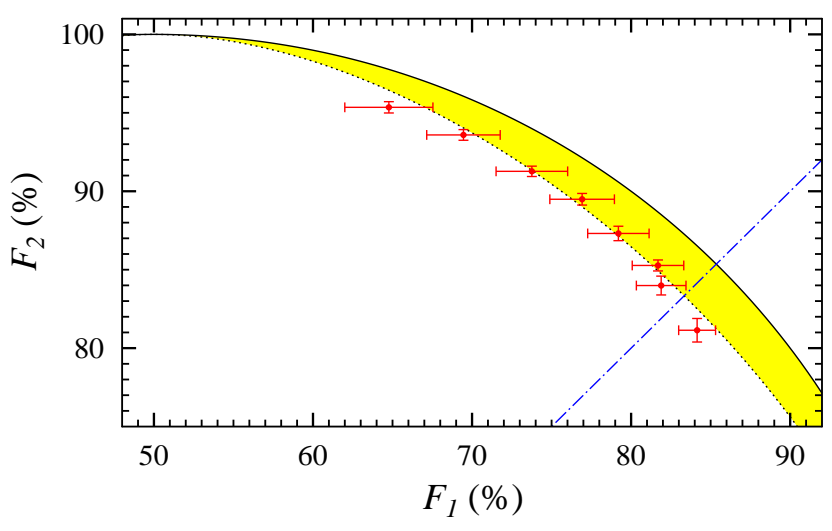

FIG. 3: (Color online) Fidelities $F_{2}$ vs $F_{1}$ of clones measured with the setup based on the special beam splitter and polarization dependent losses. The full line denotes the theoretical limit for the fidelities of the phase-covariant cloner, the dotted line shows the limit of the universal cloner. The dashed line shows the symmetric line $\left(F_{1}=F_{2}\right)$.

\begin{tabular}{cccccc}
\hline$q$ & $\Sigma_{\nu}^{-1}$ & $\Sigma_{\eta}$ & $F_{1}[\%]$ & $F_{2}[\%]$ & $P_{\text {succ }}^{\mathrm{SBS}}[\%]$ \\
\hline \hline 0.93 & 0.10 & 0.55 & $64.8 \pm 2.8$ & $95.4 \pm 0.4$ & $2.9 \pm 0.1$ \\
0.85 & 0.20 & 0.60 & $69.5 \pm 2.3$ & $93.6 \pm 0.3$ & $10.3 \pm 0.1$ \\
0.78 & 0.30 & 0.66 & $73.8 \pm 2.3$ & $91.3 \pm 0.3$ & $13.8 \pm 0.1$ \\
0.70 & 0.40 & 0.73 & $76.9 \pm 2.0$ & $89.5 \pm 0.4$ & $17.4 \pm 0.2$ \\
0.63 & 0.50 & 0.81 & $79.2 \pm 1.9$ & $87.3 \pm 0.5$ & $18.8 \pm 0.2$ \\
0.55 & 0.60 & 0.92 & $81.7 \pm 1.6$ & $85.3 \pm 0.4$ & $20.0 \pm 0.2$ \\
0.51 & 0.66 & 1.00 & $81.9 \pm 1.6$ & $84.0 \pm 0.6$ & $23.6 \pm 0.2$ \\
- & - & - & $84.2 \pm 1.2$ & $81.1 \pm 0.8$ & $28.8 \pm 0.2$ \\
\hline
\end{tabular}

TABLE I: Table of the setting of glass plates, measured fidelities and $P_{\text {succ }}^{\mathrm{SBS}}$ for the specified asymmetry parameter $q$ for the setup based on the SBS. The last row represents the measurement without glass plates.

(second) clone onto the input state and the sum of all coincidences $C_{\text {sum }}=C^{++}+C^{+-}+C^{-+}+C^{--}$,

$$
F_{1}=\frac{C^{++}+C^{+-}}{C_{\text {sum }}}, \quad F_{2}=\frac{C^{++}+C^{-+}}{C_{\text {sum }}} .
$$

The probability of success of the device is determined as a fraction of the sum of all measured coincidences to the total number of the photon pairs entering the cloner $C_{\mathrm{tot}}$, $P_{\text {succ }}=C_{\text {sum }} / C_{\text {tot }}$.

We selected seven representative values of asymmetry parameter $q$ and set the angles of the $\mathrm{GP}_{\eta}$ and $\mathrm{GP}_{\nu}$ accordingly. Then we measured clone fidelities for a set of nine states $\frac{1}{\sqrt{2}}\left(|V\rangle+e^{i k \pi / 4}|H\rangle\right), k=-4, \ldots, 4$, located on the equator of the Bloch sphere. These states span over circular and diagonal linear polarization states. Resulting mean fidelities averaged over the nine states are plotted in Fig. 3 and listed in Tab. I. The statistical errors were calculated from 10 ten-second measurements. They reach values $\sim 1 \%$ in the symmetric case. For higher degrees of asymmetry, the polarization filtration resulted in higher losses, leading to decrease of the success probability and increase of statistical errors of fidelities up to $\sim 3 \%$. The last row of Tab. Irepresents the measurement without glass plates. It shows the intrinsic asymmetric operation of the SBS. The use of the polarization filters enables to approach the symmetric operation and to go further to tune the asymmetry up to markedly asymmetric cloning.

The statistical error of mean fidelity $F_{1}$ is greater than the error of $F_{2}$. This is due to more pronounced oscillations of $F_{1}$ when scanning over the equatorial states. This effect is caused by residual uncompensated phase shifts induced by the special beam splitter. The reflected and transmitted photons acquire different phase shifts. Figure 3 shows the comparison of our measurements with the theoretical limits of the universal asymmetric cloner (5) and of the phase-covariant asymmetric cloner (4). All measured points are very close to the universal asymmetric cloning limit but do not reach the theoretical phasecovariant cloning limit on fidelity. The main effect that reduces the fidelity of the two clones and prevents us to surpass the universal cloning limit is the non-ideal overlap of the spatial modes of the two photons on the SBS.

\section{HYBRID FREE-SPACE AND FIBER SETUP}

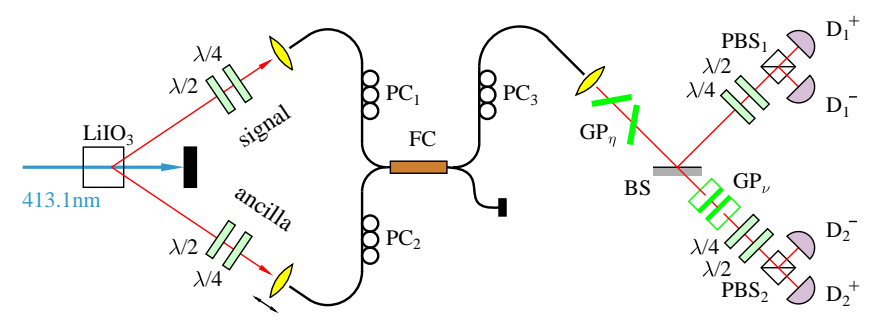

FIG. 4: (Color online) Scheme of the hybrid cloning setup. FC - fiber coupler, BS - nonpolarizing beam splitter, PBS - polarizing beam splitter, $\mathrm{PC}$ - polarization controller, $\lambda / 2, \lambda / 4$ - wave plates, $\mathrm{GP}_{\eta}, \mathrm{GP}_{\nu}$ - polarization dependent losses, D detector.

In order to increase the cloning fidelity we have built an alternative setup which combines advantages of both fiber and free space propagation, see Fig. 4. Fiber coupler (FC) ensures perfect overlap of spatial modes of signal and ancilla photons. The free space part allows to use simple encoding of information into the polarization states of the photons. We can use wave plates $(\lambda / 2, \lambda / 4)$ and polarizing beam splitters to prepare arbitrary input polarization states and to perform projective measurements in arbitrary basis. This experimental scheme is based on the universal cloner, i.e. interference of two photons on a balanced beam splitter [18, 19, 20], which is modified by the state filtering. The polarization filters $\mathrm{GP}_{\eta}$ and $\mathrm{GP}_{\nu}$ ensure implementation of phase-covariant cloning transformation, compensate for non-ideal splitting ratio of the BS and allow to tune the asymmetry 


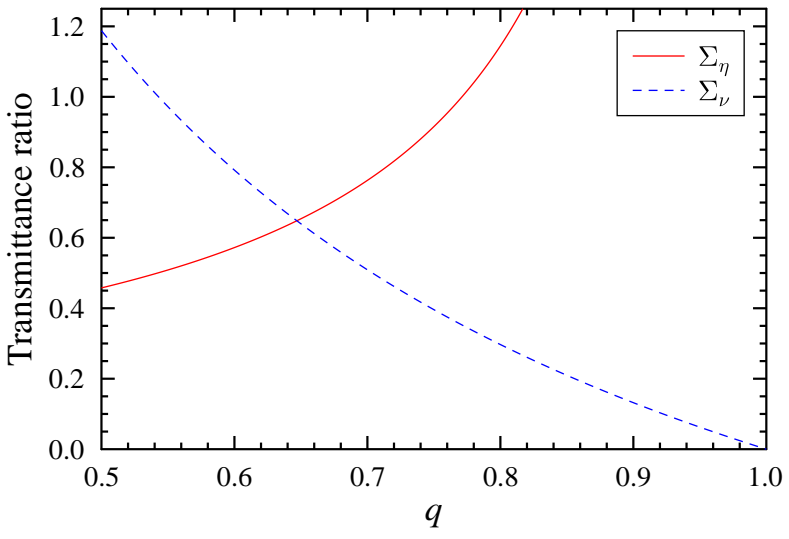

FIG. 5: (Color online) Transmittance ratios $\Sigma_{\eta}$ and $\Sigma_{\nu}$ for hybrid asymmetric cloning setup. Plotted dependences were calculated according to Eq. (13) using experimentally determined parameters of BS: $R_{V}=50.9 \%$ and $R_{H}=46.6 \%$.

of the cloner. The device again operates in the coincidence basis and the cloning is successfully accomplished if a single photon is detected in each output port of the cloner. The transformation realized by the whole device can be written as,

$$
\begin{aligned}
|V\rangle_{\text {sig }}|V\rangle_{\text {anc }} \rightarrow & 2 r t \eta_{V}^{2} t_{V} r_{V} \nu_{V}|V\rangle_{1}|V\rangle_{2}, \\
|H\rangle_{\text {sig }}|V\rangle_{\text {anc }} \rightarrow & r t \eta_{V} \eta_{H}\left(t_{V} r_{H} \nu_{V}|H\rangle_{1}|V\rangle_{2}\right. \\
& \left.+t_{H} r_{V} \nu_{H}|V\rangle_{1}|H\rangle_{2}\right),
\end{aligned}
$$

where coefficients $r$ and $t$ represent reflectance and transmittance of the FC; $r_{j}$ and $t_{j}, j=V, H$, denote reflectance and transmittance of the BS, which are slightly polarization dependent $\left(R_{V}=50.9 \%\right.$ and $\left.R_{H}=46.6 \%\right)$.

Similarly as for the previous scheme, Eq. (11) becomes equivalent to the target unitary cloning transformation (3) if the following relations hold,

$$
\begin{aligned}
& \eta_{V} \eta_{H} t_{V} r_{H} \nu_{V}=\sqrt{1-q} 2 \eta_{V}^{2} t_{V} r_{V} \nu_{V}, \\
& \eta_{V} \eta_{H} t_{H} r_{V} \nu_{H}=\sqrt{q} 2 \eta_{V}^{2} t_{V} r_{V} \nu_{V} .
\end{aligned}
$$

After some algebra we arrive at the dependence of the transmittance ratios of the polarization filters on the setup parameters and the asymmetry parameter $q$,

$$
\Sigma_{\eta}=\frac{R_{H}}{R_{V}} \frac{1}{4(1-q)}, \quad \Sigma_{\nu}=\frac{R_{V}\left(1-R_{H}\right)}{R_{H}\left(1-R_{V}\right)} \frac{1-q}{q} .
$$

Dependences of $\Sigma_{\eta}$ and $\Sigma_{\nu}$ on $q$ calculated for the parameters of our setup are plotted in Fig 5

The measurement routine starts with an adjustment of the HOM interference dip in the fiber coupler FC. In this preliminary stage two outputs of the FC are connected directly to the detectors and the overlap of the two photons is maximized finding a minimum of the coincidence counts. Optimal overlap of the polarization states on the $\mathrm{FC}$ is achieved by adjusting polarization controllers $\mathrm{PC}_{1}$ and $\mathrm{PC}_{2}$. Then one output of the $\mathrm{FC}$ is directed

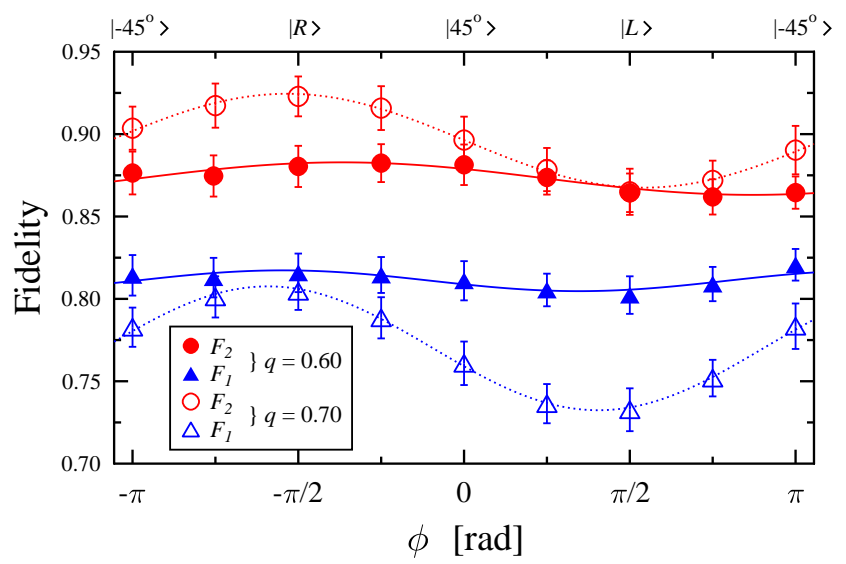

FIG. 6: (Color online) Fidelities $F_{1}$ and $F_{2}$ of cloning of equatorial qubits with the hybrid cloning setup for two asymmetry parameters, $q=0.60$ and $q=0.70$. The top axis shows the signal qubit state corresponding to the phase $\phi$ (see Eq. (2)).

to the free space part of the setup. The last polarization controller $\mathrm{PC}_{3}$ is used to compensate polarization transformation induced in the fibers. The BS splits the photon pair into two paths with probability $\frac{1}{2}$. $\mathrm{GP}_{\eta}$ and $\mathrm{GP}_{\nu}$ are tilted to provide demanded polarization state filtration. Input polarization states and measurement bases are set by half- and quarter-wave plates.

As in the previous section we performed cloning of nine polarization states of a signal qubit distributed over the equator of the Bloch sphere. Figure 6 shows two typical examples of the experimentally measured fidelities for the equatorial qubits ( $q=0.60$ not oscillating, $q=0.70$ the most oscillating one). Statistical error bars were determined from 10 twenty second measurement periods. Due to the fact, that the oscillations have the sinusoidal character and for both fidelities the sinusoids have the same phase, we suppose that we did not set the ancilla photon polarization exactly on the pole of the Bloch sphere. Higher oscillations lead to greater errors of the mean fidelities.

Note that any cloner can be converted by a twirling operation [2] to a truly phase-covariant cloner whose cloning fidelity does not depend on the input state and is equal to the mean fidelity of the original cloner. The twirling consists in application of the random phase shift operation $U(\vartheta)=|V\rangle\left\langle V\left|+e^{i \vartheta}\right| H\right\rangle\langle H|$ to the input state and the inverse operation $U(-\vartheta)$ to each of the clones. The phase shift $\vartheta$ is selected randomly from the interval $[0,2 \pi]$. In the present implementation, the twirling could be performed by using additional wave-plates.

The relevant parameters of the cloner are thus the mean cloning fidelities which fully quantify its performance. The mean fidelities are shown in Fig. 7 and are also listed in Table I As can be seen the resulting mean fidelities are above the universal cloning limit for all asymmetries. Note that due to technical limitations 


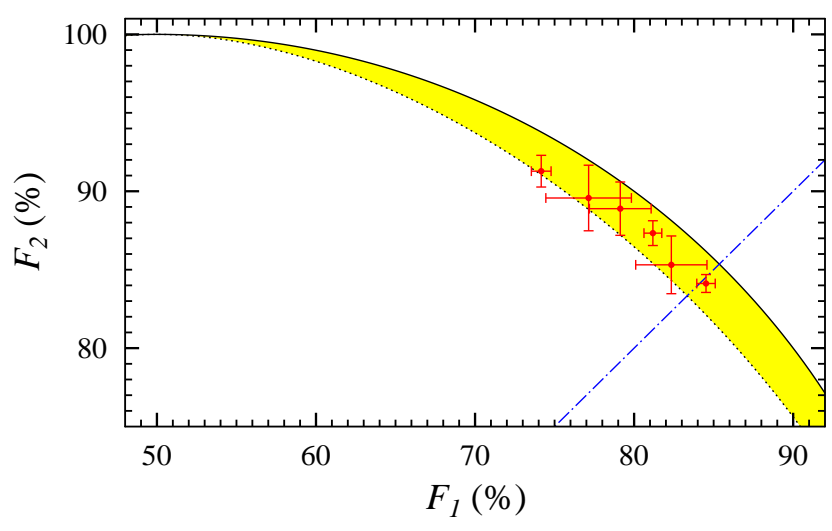

FIG. 7: (Color online) Fidelities $F_{2}$ vs $F_{1}$ of clones measured with the hybrid setup. The full line denotes the theoretical limit for the fidelities of the phase-covariant cloner, the dotted line shows the limit of the universal cloner. The dashed line shows the symmetric line $\left(F_{1}=F_{2}\right)$.

\begin{tabular}{cccccc}
\hline$q$ & $\Sigma_{\eta}$ & $\Sigma_{\nu}$ & $F_{1}[\%]$ & $F_{2}[\%]$ & $P_{\text {succ }}^{\mathrm{Hyb}}[\%]$ \\
\hline \hline 0.75 & 1.00 & 0.39 & $74.2 \pm 0.6$ & $91.3 \pm 1.0$ & $6.2 \pm 0.2$ \\
0.70 & 0.83 & 0.50 & $77.1 \pm 2.7$ & $89.6 \pm 2.1$ & $4.8 \pm 0.2$ \\
0.65 & 0.71 & 0.63 & $79.1 \pm 1.9$ & $88.9 \pm 1.7$ & $4.8 \pm 0.1$ \\
0.60 & 0.63 & 0.78 & $81.2 \pm 0.6$ & $87.3 \pm 0.8$ & $5.0 \pm 0.1$ \\
0.55 & 0.56 & 0.96 & $82.3 \pm 2.2$ & $85.3 \pm 1.8$ & $4.4 \pm 0.2$ \\
0.50 & 0.50 & 1.17 & $84.5 \pm 0.6$ & $84.1 \pm 0.6$ & $4.2 \pm 0.1$ \\
\hline
\end{tabular}

TABLE II: Table of the setting of glass plates, measured fidelities and $P_{\text {succ }}^{\mathrm{Hyb}}$ for the specified asymmetry parameter $q$ of the hybrid setup.

on achievable $\Sigma_{\eta}$ and $\Sigma_{\nu}$ we can reach only moderate asymmetries $q \in[0.50,0.75]$.

From the relations (11) we can also determine the probability of success of the hybrid setup, $P_{\text {succ }}^{\mathrm{Hyb}}=$ $\left(2 r t \eta_{V}^{2} t_{V} r_{V} \nu_{V}\right)^{2}$. For ideal symmetric cloner we obtain $P_{\text {succ }}^{\mathrm{Hyb}}=\frac{1}{16}$ which should be compared with $P_{\text {succ }}^{\mathrm{SBS}}=\frac{1}{3}$ achieved by the setup discussed in Sec. III The hybrid setup exhibits lower probability of success mainly because there are two post-selection steps. First, the signal and ancilla photon must leave the FC together by the selected output fiber (the upper one in Fig. 4). Second, there must be one photon in each output arm of the bulk BS. For completeness, we plot the measured probabilities of success of both cloning setups in Fig. 8

\section{CONCLUSIONS}

In this paper we described two experimental setups proposed to realize optimal asymmetric phase-covariant cloning of single-photon polarization qubits. We characterized the real experimental operation of both setups and compared their performances and limitations. The cloning is based on interference of the signal photon with

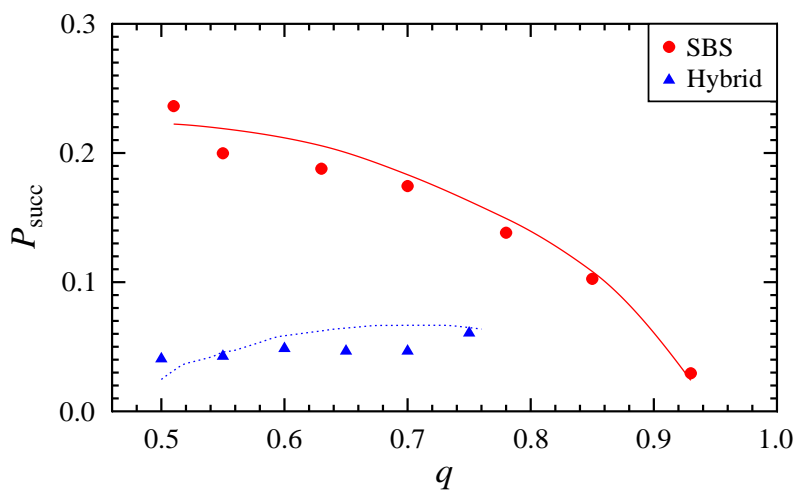

FIG. 8: (Color online) Probability of success: the setup based on the special beam splitter (circles), hybrid setup (triangles). The errors are smaller than the symbols shown. The lines represent theoretical dependences calculated from measured transmittances of the tilted glass plates.

the ancilla photon on a beam splitter or fiber coupler followed by polarization filtration on the outputs. The implemented cloning machines operate in the coincidence basis and a successful operation of the device is heralded by detection of a single photon in each output arm. An important feature of both experimental setups is that the polarization filtering allows to tune the asymmetry of the cloning operation. Moreover, the same polarization filtering is used to compensate imperfections of beam splitters whose splitting ratios slightly differed from the desired ones.

The first setup relies on a special unbalanced beam splitter with different transmittances for vertical and horizontal polarizations. The main advantage of this setup is that we can tune the asymmetry of cloning in a broad range. However, the imperfect overlap of the spatial modes of the photons on the bulk beam splitter limits the achievable fidelity of the clones and prevents us from surpassing the limit of optimal universal asymmetric cloning with this approach. The second setup is based on the fiber coupler ensuring practically perfect overlap of spatial modes. With this second approach we were able to achieve very high mean cloning fidelities exceeding the maximum fidelities obtainable by universal cloners. To the best of our knowledge, this is the first experiment where universal cloning limit has been surpassed for asymmetric cloning of equatorial polarization states of single photons. The price to pay for the fidelity improvement is a smaller probability of success of this latter scheme and also somewhat narrower accessible asymmetry range.

\section{Acknowledgments}

This research was supported by the projects LC06007, 1M06002 and MSM6198959213 of the Ministry of Education of the Czech Republic and by the EU project 
SECOQC (IST-2002-506813).

[1] V. Scarani, S. Iblisdir, N. Gisin, and A. Acín, Rev. Mod. Phys. 77, 1225 (2005).

[2] N.J. Cerf and J. Fiurášek, In: Progress in Optics, vol. 49, Edt. E. Wolf (Elsevier, 2006), p. 455.

[3] W.K. Wootters and W.H. Zurek, Nature (London) 299, 802 (1982); D. Dieks, Phys. Lett. 92A, 271 (1982).

[4] V. Bužek and M. Hillery, Phys. Rev. A 54, 1844 (1996).

[5] N. Gisin and S. Massar, Phys. Rev. Lett. 79, 2153 (1997).

[6] D. Bruss, A. Ekert, and C. Macchiavello, Phys. Rev. Lett. 81, 2598 (1998).

[7] R.F. Werner, Phys. Rev. A 58, 1827 (1998).

[8] C.-S. Niu and R.B. Griffiths, Phys. Rev. A 60, 2764 (1999).

[9] D. Bruß, M. Cinchetti, G. M. D'Ariano, and C. Macchiavello, Phys. Rev. A 62, 012302 (2000).

[10] G.M. D'Ariano and C. Macchiavello, Phys. Rev. A 67, 042306 (2003).

[11] J. Fiurasek, Phys. Rev. A 67, 052314 (2003).

[12] C.A. Fuchs, N. Gisin, R. B. Griffiths, C.-S. Niu, and A. Peres, Phys. Rev. A 56, 1163 (1997).

[13] N.J. Cerf, M. Bourennane, A. Karlsson, and N. Gisin, Phys. Rev. Lett. 88, 127902 (2002).

[14] A.T. Rezakhani, S. Siadatnejad, and A.H. Ghaderi, Phys. Lett. A 336, 278 (2005).

[15] L.-P. Lamoureux, N. J. Cerf, Quant. Inf. Comp. 5, 32
(2005).

[16] A. Lamas-Linares, C. Simon, J.C. Howell, and D. Bouwmeester, Science 296, 712 (2002).

[17] F. De Martini, D. Pelliccia, and F. Sciarrino, Phys. Rev. Lett. 92, 067901 (2004).

[18] M. Ricci, F. Sciarrino, C. Sias, and F. De Martini Phys. Rev. Lett. 92, 047901 (2004).

[19] W.T.M. Irvine, A.L. Linares, M. J. A. de Dood, and D. Bouwmeester, Phys. Rev. Lett. 92, 047902 (2004).

[20] I. AliKhan and J.C. Howell, Phys. Rev. A 70, 010303(R) (2004).

[21] Z. Zhao, A.-N. Zhang, X.-Q. Zhou, Y.-A. Chen, C.-Y. Lu, A. Karlsson, and J.-W. Pan, Phys. Rev. Lett. 95, 030502 (2005).

[22] F. Sciarrino and F. De Martini, Phys. Rev. A 72, 062313 (2005).

[23] A. Černoch, L. Bartůšková, J. Soubusta, M. Ježek, J. Fiurášek, M. Dušek, Phys. Rev. A 74, 042327 (2006).

[24] J. Soubusta, L. Bartůšková, A. Černoch, J. Fiurášek, M. Dušek, Phys. Rev. A 76, 042318 (2007).

[25] L. Bartůšková, M. Dušek, A. Černoch, J. Soubusta, J. Fiurášek, Phys. Rev. Lett. 99, 120505 (2007).

[26] C.K. Hong, Z.Y. Ou, and L. Mandel, Phys. Rev. Lett. 59, 2044 (1987). 